\begin{tabular}{|l|l|l|l|l} 
Revista Clío América & ISSN: 1909-941X & Vol. 8 & No. 16 & Julio - Diciembre de 2014 \\
\hline
\end{tabular}

\title{
Private equity y venture capital: Diferenciación y principales características
}

\author{
Private equity and venture capital: Differentiation and main characteristics
}

Leonel Arango Vásquez

Magister en Banca y Finanzas

Institución Universitaria ESUMER, Colombia

leonel.arango@esumer.edu.co

María Patricia Durango Gutiérrez

Magister en Administración Financiera

Institución Universitaria ESUMER, Colombia

maria.durango@esumer.edu.co

Tipología:

Articulo de Reflexión

Fecha de Recibido:

Junio 04 de 2014

Fecha de Aceptación:

Agosto 01 de 2014

Para citar este artículo:

Arango, V. L., \& Durango, G. M. (2014).

Private equity y venture

capital: diferenciación y principales

caracteristicas, Clío América, 8 (16), 173 - 184
Resumen: El propósito de este artículo es explicar, desde la teoría, dos posibles opciones de financiación que tienen las empresas cuando éstas no pueden acceder a las fuentes tradicionales. La industria del Capital Riesgo surge así como una fuente alternativa de financiación. Esta industria opera a través de vehículos especiales de inversión llamados fondos Private Equity y fondos Venture Capital. En general, los primeros invierten en compañías maduras y desarrolladas, mientras que los segundos lo hacen en empresas nacientes y pequeñas. El ciclo de financiación que proveen estos fondos se estructura en tres etapas principales: captación de recursos, inversión y desinversión. En este artículo se explica la diferencia entre los términos Private Equity y Venture Capital, así como las principales características de las etapas mencionadas.

Palabras clave: desinversión, Capital Riesgo, captación de fondos, inversión alternativa, pymes.

JEL: G24.

Abstract: The purpose of this article with a qualitative approach aims to determine, from the theory the business environment, when companies are not capable to obtain financing through traditional sources, the Private Equity industry is viewed as an alternative source of finance for those companies. This industry operates through special investment vehicles named Private Equity Funds and Venture Capital Funds. In general, the former invest in develop and mature firms, the latter focus on infant and small companies. The financial cycle that these funds supply is structured in three main phases: fundraising phase, investment phase, and divestment phase or exit. This paper explains the difference between Private Equity and Venture Capital, as well as the main characteristics of the mentioned phases; through the methodology of content analysis, which aims to continue the research project of Venture Capital Industry in Colombia.

Keywords: divestment, private equity, fundraising, alternative investment, SME's. 


\section{Introducción}

La industria del Capital Riesgo en Colombia es todavía naciente, con menos de 10 años de existencia. Pocos fondos operan en el mercado y también son pocas las aproximaciones que en la literatura académica colombiana se han hecho a esta industria. Sin embargo, en Colombia empieza a tomar gran importancia el acceso a esta fuente de financiación empresarial (distinta a las fuentes tradicionales como los bancos comerciales, los familiares y hasta en algunos casos, el círculo de amigos del empresario o fundador de la empresa), situación que se explica por factores como un marco legal que fomenta la creación de fondos de Capital Riesgo, menores restricciones a los inversores institucionales, protección a los accionistas minoritarios y unas prácticas de gobierno corporativo sólidas (LAVCA, 2013).

El acceso a la financiación empresarial es importante porque le permite a las compañías ejecutar proyectos de inversión y expansión. La primera fuente de financiación la constituyen los dueños de la compañía, pero, en muchas ocasiones, el capital que estos pueden aportar no es suficiente. Ante esta situación, las empresas se ven obligadas a buscar fuentes de financiación externas. (Irwin \& Scott, 2006) señalan que los bancos comerciales se constituyen entonces como la fuente de financiación más utilizada por las empresas, sin embargo, destacan algunas barreras a la financiación cuando se trata de empresas pequeñas y medianas: los bancos pueden percibir que la empresa no será capaz de alcanzar los objetivos, no existen suficientes colaterales que respalden la deuda, la experiencia de la empresa es mínima 0 insuficiente (track record), la estructura de la empresa no se adecua a los perfiles definidos por el banco, entre otras características que hacen que las solicitudes de préstamos 0 créditos sean consideradas por los bancos como muy riesgosas.

Como antecedente al desarrollo de este artículo, se realizó una búsqueda intensiva de artículos de investigación colombianos sobre la industria del Capital Riesgo y se encontró que son pocos los artículos que aborden esta temática de forma rigurosa. En la búsqueda encontramos algunos boletines y noticias publicadas en diarios económicos y financieros, así como en las páginas web de algunos de los fondos que operan en el país, pero esa información a través de esos canales de comunicación no tiene una difusión suficiente como para considerar que en Colombia los empresarios del sector Mipymes tienen un amplio conocimiento acerca de estos mecanismos de financiación. Este desconocimiento no es único de los empresarios colombianos, también se evidencia esa falta de conocimiento en algunos países europeos, donde además muchos empresarios sienten temor de entregar parte de sus acciones a cambio de capital (EVCA, 2013; Fundación de Estudios Financieros, 2005).

Surge así la idea principal que da origen a la elaboración de este artículo: en el ámbito empresarial colombiano, especialmente en el de las Mipymes, los términos Private Equity y Venture Capital son desconocidos, así como también lo es su término equivalente en español: Capital Riesgo. Este artículo brinda una descripción simplificada sobre algunos aspectos básicos de la industria del Capital Riesgo, con el ánimo de que exista una mayor cultura financiera en Colombia en torno a ese mecanismo de financiación de amplia utilización en otros países. Con el ánimo de ofrecer una mayor claridad en la conceptualización de algunos aspectos importantes de la industria del Capital Riesgo, este artículo da respuesta a las siguientes preguntas:

- ¿Qué diferencia existe entre el término Venture Capital y el término Private Equity?

- ¿Cuáles son las principales características de cada una de las etapas de los fondos que participan en la industria del Capital Riesgo?

La primera pregunta obedece a la necesidad de tener una clara diferenciación en cuanto al uso apropiado de ambos términos, debido a que en muchas ocasiones, ambos son usados de forma indistinta para referirse a las operaciones de financiación que se hacen a través de los fondos de Capital Riesgo, pero en la práctica de la industria, los fondos tipo Venture Capital están orientados hacia un tipo de empresas que tienen características distintas a las que tienen las empresas buscadas por los fondos tipo Private Equity. La respuesta a la segunda pregunta permitirá conocer en mayor detalle cuáles son los 
rasgos característicos de cada una de esas fases que son propias de todas las operaciones que se realizan en la industria.

Para responder las preguntas planteadas, se realizó una búsqueda exhaustiva de la literatura correspondiente, haciendo revisión de libros, páginas web institucionales y artículos de investigación publicados en revistas de alto reconocimiento en el mundo científico, con el fin de dotar las respuestas de la mayor rigurosidad posible. Una metodología más apropiada, sin duda, habría sido la utilización de fuentes primarias, como entrevistas y/o encuestas a los expertos o gestores de fondos que tienen presencia en Colombia, pero el intento de acceder a esas fuentes primarias fue infructuoso, impidiendo una real aproximación a ellas.

A continuación se presenta la metodología, algunas generalidades de la industria del Capital Riesgo. Posteriormente, se hace una diferenciación conceptual entre el término Private Equity y el término Venture Capital. Se describen además algunas características de las tres etapas del ciclo de inversión que siguen los fondos de Capital Riesgo. Finalmente, se presentan algunas conclusiones.

\section{Metodología}

El artículo tiene un carácter de diagnóstico cualitativo, orientado bajo la metodología del análisis de contenido, debido a que realiza inferencias con base en antecedentes y descripciones de la industria de Capital Riesgo en contenidos identificados en varios textos revisados previamente.

El artículo se construyó por fases, definiéndose en la primera la población objeto de estudio, que son las empresas que han recibido financiación a través de los fondos creados por la industria del Capital Riesgo. Posteriormente, se hizo la selección y análisis de poblaciones limitadas, donde la muestra estuvo constituida por empresas establecidas en países industrializados. En la siguiente fase se establecieron los rasgos diferenciadores de los vehículos de financiación de la industria, así como las principales características del proceso inversor de esos vehículos. Finalmente, en la fase de análisis y de redacción del artículo, se estudió e interpretó la información recolectada y se extrajeron algunas importantes conclusiones.

\section{Generalidades de la Industria}

Aunque el término Capital Riesgo no resulte en principio familiar, resulta llamativo saber que muchas de las empresas con las que sí existe cierta familiaridad han recibido en algún momento financiación a través de esta industria, empresas como Apple Computer, Cisco Systems, Genentech, Intel, Microsoft, Netscape, Sun Microsystems, Federal Express, Staples, Starbucks y TCBY. Esta industria ha tenido una importante expansión en algunos países como Estados Unidos, donde pasó de mover 3 billones de dólares en 1990 a cerca de 103 billones en el año 2000 (Woodward \& Hall, 2004).

Según (Silviera \& Wright, 2006), el Capital Riesgo es una fuente de financiación usada por diversas empresas cuando el acceso a las fuentes de financiación tradicionales es limitado. (Swärt, M., 2008) clasifica a la industria del Capital Riesgo dentro de la categoría de "inversiones alternativas" y señala algunas características de este tipo de inversiones: tienen una historia relativamente reciente, no son comunes en los portafolios de inversión, suelen ser ilíquidas, tienen características diferentes a las de los activos tradicionales, no suelen estar cotizadas en mercados públicos y requieren gestores (managers) con habilidades específicas.

Esta industria crea unos vehículos especiales de inversión llamados Fondos de Capital Riesgo (FCR), que permiten captar capital proveniente de inversores que no requieren liquidez ni a corto ni a mediano plazo y por lo tanto, dichos inversores suelen ser de carácter institucional, como los fondos de pensiones, las compañías aseguradoras, las grandes instituciones financieras y el gobierno (Kaupelytè \& Jankauskienè, 2009) El fondo es administrado por un gestor, que suele ser un individuo con la formación profesional y académica suficiente para hacer una adecuada administración y gestión del fondo y de las inversiones que éste realiza. Para (Koryak \& Smolarski, 2008), los gestores de los FCR son inversores profesionales que invierten en 
firmas con el objetivo fundamental de obtener una rentabilidad sobre el capital invertido. Las empresas en las que invierten estos fondos se caracterizan por ser de largo plazo, no estar listadas en bolsa y presentar riesgo. De forma similar, (Metrick, 2006), define a los FCR como intermediarios financieros que captan recursos para invertirlos directamente en las compañías que son privadas (no listadas), tomando una participación activa en la dirección de la compañía, con el fin de maximizar el retorno sobre el capital una vez se hace la desinversión y ayudando a financiar el crecimiento orgánico de esas compañías.

Los Fondos de Capital Riesgo son los vehículos a través de los que se captan los recursos que son aportados por los inversores (Limited Partners - LP), para que luego sea el gestor del fondo (General Partner - GP) quien seleccione aquellas compañías que son identificadas como posibles objetivos de inversión (target). La inversión en cada compañía es de largo plazo, normalmente de 7 a 10 años, aunque en pocas ocasiones se puede extender hasta los 12 años (Metrick, 2006). Una vez el gestor del fondo y su equipo de colaboradores han captado los recursos por parte de los inversores, inician la búsqueda de compañías objetivo que constituirán su portafolio de inversión, es decir, compañías que presentan alto potencial de crecimiento, que no estén cotizando en un mercado de valores, que actualmente pueden no estar teniendo la gestión más apropiada y por lo tanto, se espera que puedan valer mucho más en el futuro (Fundación de Estudios Financieros, 2005). (Lerner, Schoar, \& Wong, 2005) explican algunas de las razones que hacen que los inversores usen estos fondos en lugar de invertir directamente en las empresas. En primer lugar, hacer una inversión de forma directa implica tener una estrecha relación con la target, además de la capacidad de elaborar determinadas due diligence que en muchas ocasiones no es posible realizar. En segundo lugar, monitorear la target implica el uso de recursos con los que quizás no se cuenta. Finalmente, la figura de limited partner protege al inversor ante posibles responsabilidades que no podría evadir si invirtiese directamente en la compañía target.

\section{"Venture Capital" Vs "Private Equity"}

\section{Interpretaciones Académicas}

Exceptuando algunos pocos casos, la literatura ubica las operaciones tipo Venture Capital dentro de una categoría más amplia, denominada Private Equity, es decir, las operaciones de los fondos tipo Venture Capital se entienden como un caso particular de las operaciones más generales, que son las realizadas por los fondos tipo Private Equity.

A continuación, se presentan las interpretaciones de algunos autores que enmarcan las operaciones Venture Capital dentro de las operaciones Private Equity:

- (Sahu, Nath, \& Banerjee, 2009) definen el término Private Equity como una gran industria, dentro de la cual se llevan a cabo operaciones que van desde financiar empresas nacientes (Venture Capital) hasta operaciones más complejas como los Buyouts.

- (Talmor \& Vasvari, 2011) señalan que en la industria del Private Equity se identifican operaciones de inversión en compañías no listadas, compañías que pueden ser nacientes o también grandes y estables.

- (Balboa \& Martí, 2004) plantearon la existencia de un proceso de evolución entre estos dos mecanismos de financiación, donde en un primer estado se tiene la industria de fondos tipo Venture Capital (focalizados en empresas nacientes), para evolucionar luego hacia un estado más maduro con una industria de fondos tipo Private Equity.

- (Aizenman \& Kendall, 2008) explican con mayor detalle la diferencia existente entre ambos mecanismos, señalando que Venture Capital son las inversiones que se hacen en empresas que atraviesan etapas semilla (seed), arranque (start up), reciente creación (early stage) y fase de expansión (expansión stage), mientras que Private Equity son las inversiones hechas en compañías que ya tienen ingresos recurrentes, un producto bien definido y un número importante de clientes que les permitirían hacer una emisión de acciones al listarse en Bolsa. 
- (Metrick, 2006) ubica el Venture Capital dentro de una categoría más amplia denominada Private Equity.

- (Bozkaya \& Kerr, 2009) enmarcan las actividades Venture Capital y las operaciones Buyout ${ }^{1}$ dentro de lo que denominan mercados de Private Equity.

- (Jeng \& Wells, 2000) definen el término Venture Capital como uno de los tipos de inversiones dentro de un conjunto denominado Private Equity, haciendo énfasis en que esa es la interpretación para la época (año 2000) que se tenía de ambos términos en Estados Unidos, a diferencia de la interpretación dada en otros países.

- (Metrick \& Yasuda, 2011) consideran el término Private Equity como una industria, dentro de la cual identifican dos grandes subconjuntos, el segmento de operaciones tipo Venture Capital y el segmento de operaciones tipo Buyout.

Es interesante destacar como (Koryak \& Smolarski, 2008) rompen el esquema general en el que la gran industria se denomina Private Equity, toda vez que se refieren al término "Venture Capital" como una industria que puede dividirse en dos categorías: una donde los FCR invierten en compañías en etapas tempranas de desarrollo (Venture Capital) y otra donde los FCR invierten en compañías establecidas que requieren expandirse 0 reestructurarse (Private Equity). Es decir, ubican las operaciones tipo Private Equity dentro de una categoría más amplia que está representada por las operaciones tipo Venture Capital.

En general, los fondos tipo Private Equity invierten fundamentalmente en compañías establecidas 0 maduras, adquiriendo la mayoría accionaria de las mismas, mientras que los Fondos tipo Venture Capital invierten en compañías jóvenes o emergentes y sólo adquieren una parte minoritaria de las acciones (Kaplan \& Strömberg, 2008; Martí Pellón, 2006; Robbie \& Mike, 1998)

\footnotetext{
1. Es importante resaltar que en la literatura suele hacerse un símil entre Private Equity y operaciones tipo Buyout, debido a que éstas últimas son las operaciones más comunes en la industria del Private Equity, por lo que muchos autores usan ambos términos para referirse a esta industria.
}

\section{Interpretaciones Institucionales}

Las interpretaciones de las más reconocidas instituciones de la industria del Capital Riesgo a nivel mundial coinciden con la línea definida por los trabajos investigativos de tipo académico presentados previamente.

En Estados Unidos, la Asociación Nacional de Capital Riesgo (NVCA, 2014) denomina a la industria como Private Equity, dentro de la cual se encuentran tres tipos de operaciones: Venture Capital, Buyouts e inversiones tipo Mezzanine. La primera hace referencia a inversiones en compañías privadas (no listadas), jóvenes y de rápido crecimiento. Las dos últimas se refieren a compañías más maduras, en etapas de desarrollo más avanzadas. Bajo ambos esquemas de operación, Venture Capital y Private Equity, se adquieren acciones de la compañía participada, pero a diferencia de los fondos tipo Venture Capital, los fondos tipo Private Equity suelen realizar operaciones apalancadas, como los típicos Leverage Buyouts.

La Asociación Europea de Private Equity y Venture Capital (EVCA, 2013), la Asociación Británica de Private Equity y Venture Capital (BVCA, 2014), la Asociación Belga de Venture Capital y Private Equity (BVA, 2014), la Asociación Francesa de Private Equity (AFIC, 2014), la Asociación Australiana de Private Equity y Venture Capital (AVCAL, 2014), la Asociación Africana de Private Equity y Venture Capital (AVCA, 2014), la Oficina Australiana de Estadísticas (ABS, 2014), la Asociación de Venture Capital y Private Equity de Hong Kong (HKVCA, 2014), el Centro de Investigaciones de Venture Capital de Israel (IVC Research Center, 2014), la Asociación de Private Equity para la región del Oriente Medio y África del Norte (MENA PEA, s. f.) y la Asociación de Private Equity y Venture Capital de la India (IVCA, 2014), se aproximan bastante a la definición de su par estadounidense. Para ellas, Private Equity son las inversiones realizadas en compañías más estables y maduras, mientras que las inversiones tipo Venture Capital son aquellas orientadas a compañías nacientes (Start-ups) con alto potencial, que son dirigidas por emprendedores y que se basan fundamentalmente en innovación tecnológica. 
Según la EVCA, las operaciones Venture Capital pueden incluso invertir en fases tan tempranas como una idea o iniciativa de negocio. Así mismo, la BVCA también pone de manifiesto la práctica de los FCR de adquirir acciones de las compañías en las cuales participan a cambio de sus aportes de financiación.

\section{Venture Capital: Nuestra Interpretación}

Los fondos tipo Venture Capital son aquellos cuyo portafolio de inversión está constituido por un abanico de empresas que abarca desde las inmateriales, que están representadas en un plan de negocios, hasta las empresas pequeñas, de reciente creación, pudiendo invertir también en empresas que se encuentran en sus primeras fases de desarrollo, que tienen potencial de crecimiento orgánico, donde dicho crecimiento se basa fundamentalmente en la innovación aplicada en productos o servicios de alta aceptación en el mercado. Los sectores económicos preferidos por estos fondos son aquellos donde es posible competir con base en la tecnología y en los desarrollos innovadores, sectores como el farmacéutico, de software, de biotecnología, de nanotecnología y similares. Los Fondos tipo Venture Capital suelen invertir tomando una participación accionaria minoritaria en la compañía, pero asegurándose el tener cierto poder de participación en las decisiones más trascendentales de la compañía.

\section{Private Equity: Nuestra Interpretación}

Son las inversiones que realizan los Fondos de Capital Riesgo en compañías no listadas, que ya pasaron por la etapa de arranque, que han logrado permanecer en el mercado y consolidarse, obteniendo beneficios recurrentes y cuyas proyecciones arrojan resultados positivos y con tendencia creciente. Estas inversiones suelen ser más complejas que las realizadas por un fondo tipo Venture Capital, pues buscan darle un cambio estratégico a la compañía a través de fusiones, adquisiciones, reestructuración y operaciones similares. Los fondos tipo Private Equity suelen invertir tomando el control político de la compañía, adquiriendo una parte representativa de las acciones (mayorías).

\section{El ciclo de financiación de los fondos de capital riesgo}

\author{
Etapa De Captación De Recursos \\ (Fundraising)
}

Los recursos que capta el fondo para destinarlos a sus inversiones no son sólo importantes porque permiten sostener y financiar el proceso de expansión de las compañías invertidas, son también necesarios para prolongar la vida misma del fondo, pues ésta depende fundamentalmente de la capacidad que tenga el gestor de captar recursos en el futuro (Chung, Sensoy, Stern, \& Weisbach, 2012)"propert ies":: "formattedCitation":"(Chung, Sensoy, Stern, \& Weisbach, 2012.

La financiación que proveen los inversores a los FCR suele hacerse por etapas, que en el lenguaje propio del sector se denominan "rondas de financiación". Según (Balboa \& Martí, 2007)"container-title":"Journal of Business Venturing", "page":"453-480","volume":"2 2", "issue":"4","author":["family":"Balboa","given": "Marina","family":"Martí","given":"José"],"issued": "date-parts":[["2007"]]],"schema":"https://github. com/citation-style-language/schema/raw/master/ csl-citation.json", los inversores institucionales condicionan la aportación de recursos atendiendo a criterios como la experiencia del gestor del Fondo, la reputación del gestor en la industria y finalmente, la cantidad de recursos que haya captado previamente. Es decir, el track-record del gestor del Fondo es determinante al momento de captar nuevos recursos, bien sea de actuales inversores o de nuevos.

(Gompers \& Lerner, 1999) identifican otros factores determinantes para la fase de captación de recursos, como la reputación y el comportamiento histórico del Fondo, y destacan otro factor importante: la relación entre la oferta y la demanda en la industria del Capital Riesgo. Estos autores, con base en Poterba (1989), entienden la oferta como el deseo de los Fondos de hacer nuevas inversiones, mientras que la demanda es el deseo de las compañías de captar recursos a través de esos Fondos. Uno de los aspectos importantes de su análisis es el impuesto sobre los 
beneficios. Por el lado de la oferta, disminuciones en el impuesto sobre las ganancias de capital podrían llevar a que aumentase la disposición de los Fondos a hacer más o nuevas inversiones $y$, por el lado de la demanda, se generaría en algunos individuos (empleados de la target) el deseo de convertirse en empresarios, para intentar captar recursos a través de estas fuentes de financiación. La demanda de Capital Riesgo también se ve afectada por el nivel de crecimiento económico, aumentando cuando éste presenta una tendencia alcista.

Típicamente, los fondos de Capital Riesgo captan recursos siguiendo una secuencia de dos fases. En una primera, se obtiene el compromiso por parte de los inversores para aportar recursos al fondo y en la segunda, cuando el fondo ha identificado las compañías target atractivas, se les solicita a estos inversores que hagan el respectivo aporte, aunque no en un $100 \%$. Ellos deben realizar un primer desembolso para ejecutar las primeras inversiones y luego, pasado cierto tiempo, se les solicita el importe restante para realizar nuevas inversiones o para inyectar recursos frescos a las previamente realizadas (Cumming, Fleming, \& Johan, 2011). El proceso de financiación de las compañías bajo este esquema de rondas de financiación, le permite a los inversores decidir en cada nueva ronda si invertir nuevamente 0 no, atendiendo al comportamiento que haya presentado la compañía invertida desde la última ronda en la que se inyectó capital (Ferrary, 2010). No solo las rondas de financiación le permiten al inversor decidir si participar nuevamente basándose en el resultado de la compañía, la experiencia misma de la empresa invertida parece favorecer el proceso de captación de recursos. (Bottazzi, Da Rin, \& Hellmann, 2011) sostienen que los FCR pueden tomar un papel más activo en la compañía cuando ésta tiene un mayor nivel de experiencia. Ese activismo se ve reflejado en actividades de contratación de personal (incluyendo directivos) y en la mayor captación de recursos para ser aportados a la compañía.

Los Fondos, en su proceso natural de captación de recursos, buscan atraer nuevos inversores para que aporten capital, aunque en algunas ocasiones, esa búsqueda no se hace necesaria. Según (Ivashina \& Kovner, 2011) las futuras rondas de financiación pueden ser ejecutadas por un mismo inversor con el cual el Fondo tiene una relación previa. Esto es así debido a que con cada operación de financiación que el inversor ha realizado en el Fondo, se adquiere un mayor conocimiento sobre la manera en que opera el Fondo y de esta forma, se reduce cada vez la asimetría de información existente entre ambos desde un primer momento. El mayor conocimiento del Fondo por parte del inversor minimiza el tiempo que este último debe dedicar al monitoreo de las operaciones del Fondo.

El éxito alcanzado por el FCR en operaciones previas, las cuales le han reportado a los inversores rentabilidades importantes, explica la permanencia de un inversor en un mismo Fondo. Mayores rentabilidades generan una mayor satisfacción y confianza en el comportamiento futuro del Fondo, lo que de alguna manera "fideliza" al inversor y lo lleva a comprometerse en futuras rondas de financiación. Esto se aplica incluso en el caso en que los futuros proyectos sean de mayor tamaño, lo que implica que el capital a ser aportado por el inversor será también mayor (Jo, 2008). Otros autores como (Marti \& Balboa, 2001) señalan también que la buena reputación del fondo influye positivamente en la captación de recursos.

Es importante señalar que, según el país que se esté analizando, determinados inversores institucionales tienen mayor o menor peso en los capitales aportados a los FCR, lo que sugiere que en la industria a nivel global no existe un único tipo de inversor que sea el más representativo. En un estudio realizado por (Mayer, Schoors, \& Yafeh, 2005), se analizaron las fuentes de financiación de FCR que operaban en Alemania, Israel, Japón y Reino Unido, encontrando que las principales fuentes en cada país eran, respectivamente, los bancos, las corporaciones, las compañías de seguros y los fondos de pensiones.

Finalmente, es común encontrar en esta industria operaciones de financiación donde se unen varios inversores para inyectar a un Fondo la cantidad de dinero requerido para ejecutar una o varias operaciones de inversión. A estas transacciones se les denomina financiaciones sindicadas, donde los inversores se unen formando un "sindicato" para 
aportar recursos al Fondo. Generalmente, uno de los inversores actúa como el "líder" y los restantes como "seguidores" (Ferrary, 2010).

\section{Etapa De Inversión (Investment)}

Los fondos analizan algunas variables para determinar cuáles empresas pueden hacer parte de su portafolio de inversión. Entre esas variables se encuentra la Junta Directiva de la compañía, la cual es determinante al momento de cerrar una operación de financiación, debido a que un proceso de negociación de larga duración no es deseado por ninguna de las partes, toda vez que se genera un desgaste para ambas y unos costos extra que pueden hacer inviable la transacción. En este sentido, según (Stuart \& Yim, 2010)"container-title":"Journal of Financial Economics","page":"174-189","volume": "97","issue":"1", "author":["family":"Stuart", "give n":"Toby E.", "family":"Yim", "given":"Soojin"],"iss ued": "date-parts":[["2010"]]],"schema":"https:// github.com/citation-style-language/schema/raw/ master/csl-citation.json", una nueva ola de transacciones de tipo take-private ${ }^{2}$ se viene presentando en Estados Unidos desde 2007-2008, caracterizada por "friendly deals" (transacciones amigables), donde a diferencia de las operaciones hostiles comunes en la década de los $80^{\circ} \mathrm{s}$, ahora los directivos de la target tienen gran influencia en la negociación y en el cierre exitoso de una operación.

Además de la Junta Directiva de la compañía target, el esquema de regulación laboral del país es también determinante para los FCR cuando deciden invertir. (Bozkaya \& Kerr, 2009) encontraron evidencia al respecto al realizar algunas entrevistas a profesionales de la industria de Private Equity en diez países europeos. Las respuestas de estos profesionales mostraron que la regulación laboral determina el desarrollo de los fondos tipo Venture Capital y Private Equity. Muchos de los entrevistados manifestaron que ese es el primer aspecto que evalúan al momento de invertir y en algunos casos, a pesar de la existencia de una fuerte regulación que podría desincentivar su participación, estarían dispuestos a invertir si encuentran otras ventajas, como una mano de obra altamente calificada.

2. Operaciones que realizan los Fondos de Capital Riesgo adquiriendo la mayoría accionaria de una compañía que cotiza en Bolsa y así, sacándola de ese mercado, la convierten en una compañía privada, sobre la cual pueden tener control y gestión.
Otro factor clave en la etapa de inversión es la relación entre el gestor y la empresa invertida, debido a que esa relación no se limita sólo a un aspecto económico, de hecho, debido a que es el empresario quien conoce los más pequeños pormenores de su empresa y no así el gestor, se presenta un problema de asimetría en la información. Esa asimetría en la información se eliminaría si la relación entre ambas partes se basara completamente en la confianza y transparencia, pero este tipo de relación "perfecta" no existe. Para mitigar esta problemática, (Bonnet \& Wirtz, 2012) sugieren la posibilidad de realizar un monitoreo permanente a la empresa, lo que permitiría reducir dicha asimetría, sin embargo, esta práctica demandaría tiempo y recursos por parte de los gestores, haciendo su ejecución poco probable. (Cumming, Fleming \& Johan 2011) reafirman esta situación cuando plantean que el proceso inversor de los FCR requiere de mucha información y de altos costos de búsqueda de aquellas oportunidades atractivas para el fondo, cuando se comparan con alternativas de inversión (compañías targets) que se encuentran listadas.

Para construir el portafolio de compañías invertidas, el Fondo define previamente su filosofía de inversión, lo que hace posible acotar de forma muy precisa el tipo de inversiones viables, de manera similar a como lo hacen los fondos comunes de inversión. Sin embargo, en ese proceso de selección de compañías, el criterio del gestor del fondo es crucial. Los gestores suelen tener una formación académica y profesional intensiva y por lo tanto, suelen guiarse por una evaluación técnica de la compañía (due diligence) y por otras variables de carácter operativo (Bonnet \& Wirtz, 2012), mientras que en otras operaciones de financiación suele importar más el criterio personal ${ }^{3}$.

\section{Etapa De Desinversión (Disinvestment / Exit)}

Desinversión son todas aquellas actividades que le permiten al gestor del fondo vender el $100 \%$ o una parte de las acciones que tiene en su portafolio de compañías invertidas. Los fondos que operan en la industria del Capital Riesgo aportan recursos a

\footnotetext{
3. Algunos inversores, especialmente aquellos implicados en operaciones tipo Venture Capital, suelen guiarse más por su intuición y por la buena relación establecida (good feeling) desde el primer momento con el empresario. (Fairchild, 2011) trata este y otros temas relacionados.
} 
la empresa target a cambio de obtener una participación accionaria en ella, para de esta forma poder participar en su dirección y administración y posteriormente venderla (Gompers \& Lerner, 1999; McNeil, 2012). Al vender la empresa (operación de desinversión) pueden generarse pérdidas o beneficios. Como en cualquier otra industria, no todas las operaciones son exitosas, así, esta circunstancia explica el por qué a esta fuente de financiación se le denomina "Capital Riesgo" (Fang, Ivashina, \& Lerner, 2013). Por estas razones, la operación de desinversión, además de ser ilíquida y de largo plazo, es de vital transcendencia en esta industria y juega un papel determinante desde el mismo momento en que los Fondos inician el estudio de posibles empresas objetivo (Schwienbacher, 2002; Talmor \& Vasvari, 2011)2002; Talmor \& Vasvari, 2011.

Los inversores institucionales, como cualquier otro inversor típico, usan medidas que les permiten evaluar tanto el desempeño de la inversión como el desempeño del gestor. El mecanismo de desinversión es una de esas medidas usadas por los inversores del Fondo para medir el desempeño del gestor, debido a que con base en dicho desempeño, éstos tomarán la decisión de financiar en el futuro nuevas inversiones que quiera realizar ese gestor. Esto es así porque un buen desempeño del gestor se corresponde con tasas de retorno elevadas y positivas, en caso contrario, la inversión no sería exitosa (Gorton \& Winton, 2003). Sin embargo, la evidencia muestra que el éxito de una operación de desinversión no depende sólo del gestor. El nivel de desarrollo o madurez del mercado de capitales es determinante para el éxito de la desinversión. Un mercado de capitales maduro incrementa la posibilidad de realizar desinversiones a través de las Ofertas Públicas de Adquisición. En este sentido, cuando aumenta la rentabilidad para el FCR a través de operaciones de salida a Bolsa, aumenta también la confianza en los actuales y potenciales inversores, haciendo que se incremente también la probabilidad de obtener nuevos recursos (Dore, 2013; Gompers \& Lerner, 1999; Jo, 2008).

Los fondos de Capital Riesgo cuentan con diversos mecanismos de desinversión, los cuáles son elegidos fundamentalmente teniendo en cuenta las condiciones de mercado existentes en el momen- to de la transacción y la potencialidad que éstos puedan tener para maximizar la rentabilidad en la operación, debido a que tanto los gestores como los inversores esperan obtener el mayor beneficio posible en cada una de las operaciones que se dan por terminadas. (Talmor \& Vasvari, 2011) hacen una completa descripción de los seis principales mecanismos de desinversión que suelen elegir los gestores de los FCR. A continuación presentamos de forma simplificada cada uno de ellos:

\section{- Venta a un comprador estratégico (Trade sale):} es el mecanismo de desinversión más usado. Consiste en la venta de la totalidad de las acciones o de activos de la compañía a un comprador que hace parte de la misma industria a la que pertenece la empresa que se está vendiendo. Por esta razón, estas operaciones suelen ser de tipo adquisición (adquisition). Los FCR prefieren esta modalidad de desinversión porque les permite tener un mayor control sobre el proceso mismo, debido a que sólo deben negociar con un único comprador, haciendo que la negociación sea más rápida y eficiente.

- Oferta pública de adquisición (IP0): es una de las formas de desinversión más deseada por los gestores de los FCR, debido a que con ella se maximiza la rentabilidad. Sin embargo, no todas las transacciones puede hacerse a través de esta vía, debido a que la posibilidad de listarse en Bolsa está reservada sólo para compañías muy consolidadas, que tengan un valor de mercado mínimo, que cumplan con una serie de condiciones que son establecidas por la Bolsa en la que se quieran inscribir, que tenga controles internos estrictos en términos financieros, legales y contables, entre otras condiciones.

- Venta a otro fondo de Capital Riesgo (Secondary Buyout): este tipo de operación implica la venta de la compañía a otro fondo de Capital Riesgo. Este mecanismo de desinversión es especialmente útil porque le permite al fondo adquiriente aportar su expertise para que la compañía adquirida pueda avanzar hacia una etapa más madura de su desarrollo. Las operaciones tipo secondary buyout suelen ser una importante salida a la inversión en momentos en que el costo del apalancamiento financiero es bajo, puesto que hace posible que 
algunos FCR se apalanquen para comprar compañías que son de su interés y que hacen parte del portafolio de inversión de otro fondo.

- Recapitalización (Recapitalization): una operación de recapitalización consiste en la recompra de acciones a través del endeudamiento (leverage), por ejemplo, emitiendo bonos. Este mecanismo de desinversión es idóneo para muchos fondos que quieren evitar el pago de impuestos derivados de los dividendos, de tal forma que al intercambiar acciones por deuda, buscan refugiarse bajo el escudo fiscal que representa la deuda, debido a que en la mayoría de los esquemas impositivos, los intereses son deducibles.

- Recompra de acciones (Share repurchase): se presenta cuando la compañía compra las acciones que posee el FCR. La posibilidad de que esta clase de operaciones ocurran depende, en muchas ocasiones, de lo que se pacte desde el mismo momento en que el fondo entra a participar en la compañía. Existen, por ejemplo, clausulas tipo Buyback rights, que obligan a los dueños de la compañía o a su equipo directivo a comprar las acciones que posee el fondo. Otra cláusula del tipo Put Option Rights, obliga a la empresa a comprar las acciones a un precio fijado previamente, si así lo exige el fondo en el momento de la desinversión.

- Separación y liquidación (Breakups and liquidations): este es el mecanismo menos preferido por los fondos de Capital Riesgo, debido a que no se espera que la empresa termine en alguna de estas situaciones. Una situación de liquidación se presenta cuando la compañía es incapaz de sostener su deuda bancaria y por esa razón, es el correspondiente banco quien entra a tomar control sobre la misma. Una situación de separación se presenta cuando se requiere dividir la compañía en negocios independientes y venderlos posteriormente a compradores estratégicos. En ocasiones, esta venta individualizada se ve reflejada en un mayor valor que si se vendiera la empresa como un todo.

\section{Conclusiones}

- Los fondos de Capital Riesgo aportan recursos a cambio de tomar un lugar en la dirección de la compañía, con el fin de poder participar en la toma de decisiones y en la definición de políticas y estrategias que ayuden a la compañía a crecer en el mediano y largo plazo.

- Los inversores que aportan recursos a los fondos de Capital Riesgo suelen ser institucionales, como los fondos de pensiones, compañías aseguradoras, bancos de inversión, familias con mucho dinero, entre otros. Estos inversores no requieren liquidez ni a corto ni a mediano plazo, por esa razón son los candidatos naturales para proveer capital a este tipo de fondos.

- Los fondos de Capital Riesgo son dirigidos por uno o varios gestores profesionales con amplios conocimientos financieros y económicos, que son apoyados por un equipo de trabajo altamente calificado en otros temas como los contables y legales. Cada fondo define su filosofía de inversión, por esa razón pueden invertir en compañías pequeñas o nacientes, así como en compañías maduras y en etapas avanzadas de desarrollo.

- El mundo académico, así como varias instituciones en diversos países que agremian a las firmas de Capital Riesgo, ubican las operaciones tipo Venture Capital dentro de una categoría más amplia, denominada Private Equity. Específicamente, las transacciones tipo Private Equity están dirigidas a empresas maduras, con amplia experiencia en el sector y que se encuentran en una etapa de desarrollo avanzada, mientras que las de tipo Venture Capital están orientadas hacia compañías más pequeñas, que pueden ser nacientes y que tienen poca experiencia en el sector al que pertenecen.

- El proceso completo de financiación empresarial, observado en la industria del Capital Riesgo, se puede dividir en tres etapas: captación de fondos, inversión y desinversión. En la primera, inversores institucionales aportan recursos al fondo. En la segunda, el gestor del fondo selecciona las compañías objetivo y procede a realizar las correspondientes inversiones en dichas compañías. En la tercera etapa, el gestor del fondo selecciona el mecanismo de desinversión más apropiado, con el fin de maximizar la rentabilidad y de hacer menos lenta y costosa dicha transacción. 


\section{Referencias Bibliográficas}

ABS. (2014, febrero 13). Venture Capital and Later Stage Private Equity. Recuperado a partir de http://www.ausstats. abs.gov.au/ausstats/subscriber.nsf/0/28A496CE6E1095C5 CA257C7D000COE4E/\$File/56780_2012-13.pdf

AFIC. (2014). A leading industry. Recuperado a partir de http://www.afic.asso.fr/en/private-equity-in-france/aleading-industry.html

Aizenman, J., \& Kendall, J. (2008). The internationalization of venture capital and private equity. National Bureau of Economic Research.

AVCA. (2014). International Private Equity and Venture Capital Valuation Guidelines. Recuperado a partir de http://www. avca-africa.org/product/international-private-equity-andventure-capital-valuation-guidelines/

AVCAL. (2014). Statistics \& Research. Recuperado a partir de http://www.avcal.com.au/stats-research/fact-sheets

Balboa, M., \& Martí, J. (2004). From venture capital to private equity: The Spanish experience. The Journal of Private Equity, 7(2), 54-63.

Balboa, M., \& Martí, J. (2007). Factors that determine the reputation of private equity managers in developing markets. Journal of Business Venturing, 22(4), 453-480.

Bonnet, C., \& Wirtz, P. (2012). Raising capital for rapid growth in young technology ventures: when business angels and venture capitalists coinvest. Venture Capital, 14(2-3), 91-110.

Bottazzi, L., Da Rin, M., \& Hellmann, T. F. (2011). The importance of trust for investment: Evidence from venture capital. National Bureau of Economic Research.

Bozkaya, A., \& Kerr, W. R. (2009). Labor regulations and European private equity. National Bureau of Economic Research.

BVA. (2014). Private Equity. Recuperado a partir de http:// www.bva.be/fb111cggc622vjw1szu66.aspx

BVCA. (2014). BVCA Guide to Private Equity. Recuperado a partir de http://www.bvca.co.uk/ResearchPublications/ BVCAGuidetoPrivateEquity.aspx

Chung, J.-W., Sensoy, B. A., Stern, L., \& Weisbach, M. S. (2012). Pay for performance from future fund flows: the case of private equity. Review of Financial Studies, 25(11), 3259-3304.

Cumming, D., Fleming, G., \& Johan, S. A. (2011). Institutional investment in listed private equity. European Financial Management, 17(3), 594-618.

Dore, T. E. (2013). Fundraising Shocks and Efficient Investment in Venture Capital. Job Market Paper. Recuperado a partir de http://home.uchicago.edu/tdore/JMP_Fundraising\%20 Shocks\%20and\%20Efficient $\% 20$ Investment.pdf

EVCA. (2013, noviembre). Guide on Private Equity and Venture Capital for Entrepreneurs. An EVCA Special Paper. Recuperado a partir de http://www.evca.eu/uploadedfiles/ home/toolbox/introduction_tutorial/evca_pevcguide.pdf

Fairchild, R. (2011). Fairness norms and self-interest in venture capital/entrepreneur contracting and performance. International Journal of Behavioural Accounting and Finance, 2(1), 4-20.

Fang, L., Ivashina, V., \& Lerner, J. (2013). Combining banking with private equity investing. Review of Financial Studies, 26(9), 2139-2173.

Ferrary, M. (2010). Syndication of venture capital investment: the art of resource pooling. Entrepreneurship Theory and Practice, 34(5), 885-907.

Fundación de Estudios Financieros. (2005, marzo). El Ciclo del Capital Riesgo en Europa: Su gestión y Aportación de Valor. Recuperado a partir de http://www.aldoolcese.es/ sites/default/files/9.-118823838510.pdf

Gompers, P. A., \& Lerner, J. (1999). What drives venture capital fundraising? National bureau of economic research.

Gorton, G., \& Winton, A. (2003). Financial intermediation. Handbook of the Economics of Finance, 1, 431-552.

HKVCA. (2014). Venture Capital \& Private Equity. Recuperado a partir de http://web.hkvca.com.hk/en/vc-pe.aspx

Irwin, D., \& Scott, J. M. (2006). Barriers faced by SMEs in raising bank finance. En Institute for Small Business \& Entrepreneurship.

Ivashina, V., \& Kovner, A. (2011). The private equity advantage: Leveraged buyout firms and relationship banking. Review of Financial Studies, 24(7), 2462-2498.

IVC Research Center. (2014). Research Center. Financing terms. Recuperado a partir de http://www.ivc-online. com/language/en-US/Research_Center/Keyword_Glossary/ Financing_Terms.aspx

IVCA. (2014). Glossary of terms. Recuperado a partir de http:// indiavca.org/research/glossary-of-terms.html

Jeng, L. A., \& Wells, P. C. (2000). The determinants of venture capital funding: evidence across countries. Journal of corporate Finance, 6(3), 241-289.

Jo, H. (2008). WHY DO PRIVATE EQUITY FUNDS-OF-FUNDS GROW? Journal of the Academy of Business \& Economics, 8(3).

Kaplan, S. N., \& Strömberg, P. (2008). Leveraged buyouts and private equity. National Bureau of Economic Research.

Kaupelytė, D., \& Jankauskienè, V. (2009). STIMULATING PENSION FUNDS INVESTMENTS TO VENTURE CAPITAL: OPPORTUNITIES IN EUROPEAN UNION COUNTRIES. European Integration Studies, (3). 
Private equity y venture capital: Diferenciación y principales características

Koryak, 0., \& Smolarski, J. (2008). Perception of Risk by Venture Capital and Private Equity Firms: A European Perspective. The Journal of Private Equity, 11(2), 30-42.

LAVCA. (2013). Scorecard 2013. Recuperado a partir de http:// lavca.org/wp-content/uploads/2013/04/2013-LAVCAScorecard.pdf

Lerner, J., Schoar, A., \& Wong, W. (2005). Smart Institutions, Foolish Choices? The Limited Partner Performance Puzzle, NBER Working Paper 11136.

Marti, J., \& Balboa, M. (2001). Determinants of Private Equity Fund Raising in Western Europe. En Social Science Research Network Working Paper, presented at the EFMA 2001 Lugano Meetings.

Martí Pellón, J. (2006). Imperfecciones en el mercado español de capital riesgo. Perspectivas del Sistema Financiero, (86), 73-83.

Mayer, C., Schoors, K., \& Yafeh, Y. (2005). Sources of funds and investment activities of venture capital funds: evidence from Germany, Israel, Japan and the United Kingdom. Journal of Corporate Finance, 11(3), 586-608.

McNeil, B. J. (2012). Hedge Funds and Private Equity Funds as Investment Vehicles. Wolters Kluwer. Law \& Business. Recuperado a partir de http://www.dol.gov/ebsa/pdf/ mcneil071911.pdf

MENA PEA. (s. f.). The Veeceepreneur: A guide to Venture Capital in the Middle East and North Africa (for Entrepreneurs). Recuperado a partir de http://www.menapea.com/research-association.php

Metrick, A. (2006). Venture capital and the finance of innovation. John Wiley and Sons.
Metrick, A., \& Yasuda, A. (2011). Venture capital and other private equity: a survey. European Financial Management, 17(4), 619-654.

NVCA. (2014). FAQ. Recuperado a partir de http://www.nvca. org/index.php?option=com_content\&view=article\&id $=11$ 9\&Itemid $=621$

Robbie, W., \& Mike, K. (1998). Venture capital and private equity: a review and synthesis. Journal of Business Finance \& Accounting, 25(5-6), 521-570.

Sahu, R., Nath, A., \& Banerjee, P. (2009). Trends in Private Equity and Venture Capital Investments with Special Focus on the Booming India Growth Story. J. Int'l Com. L. \& Tech., 4, 128.

Schwienbacher, A. (2002). An empirical analysis of venture capital exits in Europe and the United States. En EFA 2002 Berlin Meetings Discussion Paper, University of Amsterdam.

Silviera, R., \& Wright, R. (2006). The venture capital cycle. Unpublished working paper.

Stuart, T. E., \& Yim, S. (2010). Board interlocks and the propensity to be targeted in private equity transactions. Journal of Financial Economics, 97(1), 174-189.

Swärt, M. The Rise of Infrastructure Funds - A Case Study of Macquarie's Arlanda Express Buyout (5 de junio de 2008). Recuperado a partir de http://arc.hhs.se/download. aspx?MediumId $=566$

Talmor, E., \& Vasvari, F. (2011). International private equity. John Wiley \& Sons.

Woodward, S. E., \& Hall, R. E. (2004). Benchmarking the returns to venture. National Bureau of Economic Research. 